\title{
DESENVOLVIMENTO, CARACTERIZAÇÃO DE FILMES COMESTÍVEIS DE FÉCULA DE MANGARITO (Xanthosoma mafaffa Schott) E SUA APLICAÇÃO EM FRUTOS DE JABUTICABA
}

\author{
LIONORA FRANCISCA DE OLIVEIRA* \\ DIEGO PALMIRO RAMIREZ ASCHERI ${ }^{* *}$ \\ JOSÉ LUÍS RAMÍREZ ASCHERI ${ }^{* * *}$
}

\begin{abstract}
O presente trabalho teve por objetivo utilizar a fécula dos rizomas filhos do mangarito na elaboração de coberturas comestíveis para conservação pós-colheita de frutos de jabuticaba. A qualidade da cobertura foi avaliada por meio da permeabilidade e solubilidade dos filmes biodegradáveis e também mediante parâmetros físico-químicos das frutas determinados em função do tempo de conservação. Os filmes biodegradáveis foram preparados a partir da fécula de mangarito e glicerol em concentrações que variaram de 1,44 e 3,56 g, e 12,93 e 27,70\%, respectivamente. As coberturas biodegradáveis foram elaboradas com 2,5\% de fécula e concentrações de glicerol que variaram de 10 a $30 \%$. Utilizouse o delineamento experimental central composto, tipo estrela, com cinco repetições no ponto central e quatro axiais para verificar o efeito da variação da fécula e plastificante sobre a solubilidade e a permeabilidade dos filmes, visando seleção das melhores coberturas pela metodologia de superfície de resposta. Para a conservação pós-colheita adotou-se delineamento inteiramente casualizado disposto em esquema fatorial $4 \times 5$, com cinco repetições, em que 0 primeiro fator correspondeu aos tratamentos ( 0 a $30 \%$ de glicerol) e o segundo ao tempo de armazenamento ( 0 a 4 dias). A elevação da concentração de glicerol na solução filmogênica colaborou com o aumento da permeabilidade e solubilidade dos filmes. As coberturas biodegradáveis não foram eficientes no controle das transformações físico-químicas das jabuticabas quanto a perda de massa, $\mathrm{pH}$ e relação entre os sólidos solúveis totais e a acidez total titulável, não sendo verificada diferença significativa entre os frutos revestidos ou não com as várias coberturas.
\end{abstract}

PALAVRAS-CHAVE: Xanthosoma mafaffa Schot; AMIDO; JABUTICABA; FILMES COMESTIVEIS.

* $\quad$ Mestre em Engenharia Agrícola, Unidade de Ciências Exatas e Tecnológicas (UnUCET), Universidade Estadual de Goiás (UEG), Anápolis, GO (e-mail: lionoraf@hotmail.com).

** Engenheiro de Alimentos, M.Sc. e Doutor em Engenharia de Alimentos, Docente UnUCET/UEG, Campus Henrique Santillo, Anápolis, GO, Brasil (e-mail: ascheridpr@uol.com.br).

*** Engenheiro de Alimentos, M.Sc. e Doutor em Tecnologia de Alimentos, Pesquisador da Embrapa Agroindústria de Alimentos, Guaratiba, RJ, Brasil. 


\section{INTRODUÇÃo}

O mangarito (Xanthosoma mafaffa Schott), planta herbácea sem caule aéreo, pertence à família Araceae. Originário da região centro-americana, que engloba as Américas Central e do Sul, pode ser encontrado no México, Venezuela, Colômbia, Panamá, Costa Rica, Porto Rico, Peru e Brasil (COSTA et al., 2008; LEITE et al., 2007; MONTEIRO e PERESSIN, 1997). No Brasil, populações Guarani vêm durante séculos mantendo e até mesmo gerando a biodiversidade de seus cultivares tradicionais, entre os quais o mangarito (UTERMORHL e NUNES JR, 2006). É conhecido também como tannia, tiquisque, malangay, mangará, taioba portuguesa e mangareto, sendo denominado pela população Guarani de tayaó (COSTA et al., 2008; LEITE et al., 2007; MONTEIRO e PERESSIN, 1997; UTERMORHL e NUNES JR, 2006).

O mangarito apresenta rizoma subterrâneo principal (mãe ou primário) com brotações laterais (filhos ou secundários) e várias folhas grandes brotam do rizoma principal. No entanto, poderiam ser estudados, principalmente os rizomas filhos, que devido a suas pequenas dimensões são pouco atrativos para a culinária, assim como a fécula que pode ser aplicada em filmes biodegradáveis e/ ou coberturas comestíveis.

A obtenção dos biofilmes e/ou coberturas comestíveis baseia-se na dispersão ou solubilização de polímeros naturais como, por exemplo, a fécula em solvente (água, etanol ou ácidos orgânicos) e no acréscimo de aditivos (plastificantes) que gera solução ou dispersão filmogênica (GONTARD, GUILBERT e CUQ, 1992). A sua utilização dependerá das propriedades físicas que envolvem a capacidade de conservar alimentos durante a vida-de-prateleira. Essas propriedades podem determinar, no caso dos filmes, a permeabilidade em vapor de água e a solubilidade em água. Mede-se, no caso de coberturas comestíveis, sua capacidade de conservação indiretamente por meio das propriedades inerentes ao produto a conservar. Em frutas, por exemplo, medem-se a perda de massa, pH, sólidos solúveis totais (SST), acidez titulável total (ATT) e relação SST/ATT, entre outras qualidades do produto final.

A qualidade pós-colheita de frutos está intimamente ligada ao acondicionamento da preservação, à integridade física dos produtos e diminuição das atividades de respiração e transpiração, que conservam as características intrínsecas dos frutos (FERREIRA et al., 2004). Assim, as funções da aplicação das coberturas são diminuir a migração de umidade e de gases entre o fruto e o ambiente, manter a estrutura original dos produtos e reter seus compostos aromáticos característicos. O controle da solubilidade em água, e as propriedades de barreira influenciam diretamente a estabilidade do fruto durante a sua estocagem.

O presente trabalho teve por objetivo utilizar a fécula dos rizomas filhos do mangarito na elaboração de coberturas comestíveis para conservação pós-colheita de frutos de jabuticaba. A qualidade da cobertura foi testada, inicialmente, por meio da solubilidade e permeabilidade dos filmes biodegradáveis e também mediante parâmetros físico-químicos das frutas, determinados em função do tempo de conservação.

\section{MATERIAL E MÉTODOS}

O experimento foi realizado nos Laboratórios de Química da Unidade Universitária de Ciências Exatas e Tecnológicas da Universidade Estadual de Goiás (UEG), localizada em Anápolis (Goiás). A determinação da viscosidade da pasta foi efetuada no Laboratório de Reologia da Embrapa Agroindústria de Alimentos (EMBRAPA/RJ). O período de realização compreendeu os meses de setembro de 2008 a julho de 2009.

Os rizomas filhos foram colhidos no perímetro rural do município de Uberlândia (Minas Gerais) quando suas folhas estavam secas e senescentes. Selecionaram-se rizomas sem partes danificadas ou podres, que foram embalados em sacos de ráfia (permitindo a circulação de ar) e transportados para o Laboratório de Química da UEG para a extração da fécula. 
Depois de pesados e selecionados, os rizomas filhos foram lavados em água corrente e secos em temperatura ambiente. Esses foram moídos em moinho de facas tipo "CROTON" (Marconi, MA-580, Piracicaba/SP, Brasil) até passarem por peneira de malha contendo furos de $1 \mathrm{~mm}$ de diâmetro, com água abundante. Extraiu-se a fécula contida na polpa por peneiragens sucessivas em peneiras de malhas finas com diâmetros entre 150 e 38 mm, seguidas de decantação em baldes de plástico.

A fécula extraída foi lavada com álcool etílico absoluto para remoção de substâncias gordurosas, filtrada à vácuo e seca em estufa com circulação de ar (Marconi MA-035, Piracicaba, Brasil) a $45^{\circ} \mathrm{C}$ até massa constante. Após resfriada em dessecadores (temperatura ambiente), a fécula foi acondicionada em frascos até sua utilização.

Para o preparo das soluções filmogênicas foi necessário determinar a temperatura e o tempo de gelatinização da fécula. Obtiveram-se esses parâmetros em Determinador Rápido de Viscosidade (RVA 4, Newport Scientific PTY LTD, Sydney, Austrália), segundo o método descrito por Ascheri et al. (2006). Preparou-se solução aquosa com $3 \mathrm{~g}$ de fécula (em base seca) em $25 \mathrm{~mL}$ de água destilada, a qual foi submetida à leitura no RVA. Manteve-se a temperatura inicial a $50^{\circ} \mathrm{C}$ por $1 \mathrm{~min}$, seguido de aquecimento com taxa de $6^{\circ} \mathrm{C}$ por minuto até $95^{\circ} \mathrm{C}$, permanecendo constante por $3 \mathrm{~min}$. A pasta foi resfriada até $50^{\circ} \mathrm{C} \mathrm{com}$ taxa de $6^{\circ} \mathrm{C}$ por min. $\mathrm{O}$ gráfico obtido forneceu a temperatura e o tempo de viscosidade máxima utilizados para gelatinização da fécula.

As soluções filmogênicas (SF) foram preparadas com $100 \mathrm{~g}$ de água destilada, adicionadas de glicerol e fécula de mangarito, em concentrações que variaram de acordo com a Tabela 1. Após preparadas, as soluções foram aquecidas em banho-maria na temperatura e no tempo de viscosidade máxima estabelecidos no RVA, sob agitação constante até ocorrer a total gelatinização (por 15 a 20 minutos).

\section{TABELA 1 - NÍVEIS DOS FATORES CODIFICADOS E REAIS PARA O PLANEJAMENTO EXPERIMENTAL DO PREPARO E ELABORAÇÃO DOS BIOFILMES}

\begin{tabular}{lccccc}
\hline \multirow{2}{*}{ Fator } & \multicolumn{5}{c}{ Níveis dos fatores } \\
\cline { 2 - 6 } & $-\alpha(1,414)$ & -1 & 0 & +1 & $+\alpha(1,414)$ \\
\hline Fécula $(\mathrm{g})$ & 1,0 & 1,4 & 2,5 & 3,6 & 4,0 \\
Glicerol $(\%)$ & 10,0 & 12,9 & 20,0 & 27,7 & 30,0 \\
\hline
\end{tabular}

Realizou-se a secagem em estufa com circulação de ar forçada a $30^{\circ} \mathrm{C}$ por $24 \mathrm{~h}$, usando $10 \mathrm{~mL}$ das SF ainda quentes que foram transferidas para placas de Petri de acrílico com diâmetro de $8 \mathrm{~cm}$ e acondicionadas nas bandejas da estufa.

Os filmes biodegradáveis resultantes foram armazenados em dessecadores, contendo sílica-gel à temperatura de $21 \pm 2^{\circ} \mathrm{C}$ por $24 \mathrm{~h}$, aguardando-se 3 dias para a retirada do filmes das placas Petri.

Os filmes biodegradáveis elaborados foram caracterizados quanto à permeabilidade ao vapor de água (Pva) e solubilidade em água, sendo as análises realizadas em triplicata.

Determinou-se a Pva gravimetricamente, segundo teste da ASTM (1995), modificado por Gontard, Guilbert e CUQ (1992). Os filmes, em forma de disco de 2,2 cm, foram colocados em célula contendo sílica gel (UR $=0 \%$; pressão de vapor de $0 \mathrm{~Pa}$ ), formando membrana de modo a garantir que a migração de umidade ocorresse exclusivamente através dos filmes. Colocouse a célula em sala climatizada a $22^{\circ} \mathrm{C}$ com umidade relativa e pressão de vapor constante. A célula foi pesada em balança analítica (Bioprecisa, FA2104N, São Paulo, Brasil), com precisão de $0,0001 \mathrm{~g}$, a cada $24 \mathrm{~h}$ por 9 dias consecutivos. Calculou-se a permeabilidade por meio da Equação 1: 
Em que:

$$
P v a=\frac{G V}{A T\left(p_{1}-p_{2}\right)}
$$

$\mathrm{Pva}=$ permeabilidade ao vapor de água $\left(\mathrm{g} \mathrm{m}^{-1} \mathrm{~s}^{-1} \mathrm{~Pa}^{-1}\right)$;

$\mathrm{G}=$ massa $(\mathrm{g})$ da célula durante $24 \mathrm{~h}$;

$V=$ espessura $(m)$ média do filme;

$A=$ área $\left(m^{2}\right)$ de permeação do filme;

$\mathrm{T}=$ tempo (s);

$p_{1}-p_{2}=$ gradiente de pressão $(\mathrm{Pa})$ de vapor entre as superfícies do filme $\left(0,429710^{4} \mathrm{~Pa}\right)$.

Determinou-se a solubilidade em água segundo metodologia proposta por Gontard, Guilbert e Cuq (1992), com modificações. Inicialmente registrou-se a massa seca da amostra de filme de $2 \mathrm{~cm}$ de diâmetro em função da sua umidade, determinada em estufa a $105^{\circ} \mathrm{C}$ por $24 \mathrm{~h}$. A amostra foi, então, imersa em $50 \mathrm{~mL}$ de água destilada e o sistema mantido sob agitação a $175 \mathrm{rpm}$ em mesa agitadora orbital (Tecnal, TE-420, Piracicaba, Brasil) a $25^{\circ} \mathrm{C}$ por $24 \mathrm{~h}$. Após esse período, as amostras foram retiradas da água e a massa seca determinada nas mesmas condições descritas para obtenção da massa seca inicial. A solubilidade em água, expressa em porcentagem de material solubilizado, foi calculada a partir dos resultados em triplicata por meio da Equação 2:

$$
\text { Massa }(\%)=\frac{P I-P F}{P I} \times 100
$$

Em que:

$\mathrm{PI}=$ massa inicial do material seco;

$\mathrm{PF}=$ massa do material seco não solubilizado.

Para verificar o efeito das quantidades de fécula e glicerol adicionadas na solução filmogênica sobre a solubilidade e permeabilidade dos filmes biodegradáveis, bem como para selecionar os melhores biofilmes para posterior uso em pós-colheita, usou-se delineamento central rotacional composto, com cinco repetições no ponto central e quatro axiais. As variáveis foram estabelecidas com três níveis codificados $-1,0,+1$ de acordo com Box, Hunter e Hunter (1978). Esse delineamento apresentou quatro níveis de variáveis axiais codificados como $-\alpha e+\alpha$. O valor de $\alpha$ é função do número de variáveis independentes, sendo definido como:

$$
\alpha=\left(2^{k}\right)^{1 / 4}=\left(2^{2}\right)^{1 / 4}=1,414
$$

O delineamento estatístico requer número mínimo de tratamentos experimentais. Foram usados 11 tratamentos (com três repetições), sendo quatro fatoriais (combinações dos níveis -1 e +1), quatro axiais (uma variável no nível $\pm \alpha$ e outra no nível 0 ) e cinco centrais servindo como estimativa do erro experimental para determinar a precisão do modelo polinomial (COCHRAN e COX, 1964). As respostas ( $Y$ ) ou variáveis dependentes estudadas foram: solubilidade e permeabilidade ao vapor de água. Fazendo uso da análise de variância (ANOVA), a 5\% de probabilidade, aplicou-se equação de segunda ordem para explicar a tendência das variáveis respostas, cuja equação geral de acordo com Khuri e Cornell (1987) foi:

Em que:

$$
Y=b_{0}+\sum_{i=1}^{k} b_{i} x_{i}+\sum_{i=1}^{k} b_{i} x_{i}^{2}+\sum_{i<j} b_{j} x_{i} x_{j}+\varepsilon
$$

$Y=$ função resposta genérica;

$\mathrm{x}=$ variável real;

$b=$ coeficientes estimados pelos métodos dos mínimos quadrados, sendo sua significância avaliada pelo valor da probabilidade (valor- $p$ ), adotando-se valor de $p \leq 0,05$ para todos os ensaios;

$\varepsilon=$ erro experimental.

A adequação do modelo polinomial foi avaliada comparando-se a proporção da variação explicada, isto é, pela análise do coeficiente de determinação ajustado ( $R R_{a_{j}}^{2}$ ) e pelo método 
de seleção passo à frente (forward selection) (CHARNET, 1999) até que o valor das somas dos quadrados do erro (SQE) não variasse ou até completar os coeficientes do modelo proposto. Os coeficientes da regressão foram escolhidos de acordo com os valores de $p \leq 0,05$.

Os gráficos de superfície de resposta foram desenhados por meio do modelo matemático proposto, mantendo-se a resposta em função do eixo $Z$, com eixos $X$ e $Y$ representando os fatores independentes.

Para a conservação pós-colheita utilizaram-se frutos de jabuticabas da cultivar "Sabará", em estágio de maturação completa, recém-colhidos, obtidos diretamente de produtor da região de Hidrolândia (GO/Brasil). Foram utilizados lotes homogêneos de frutas sem defeitos ou danos. Os frutos foram selecionados em função do tamanho, cor e ausência de danos, lavados em água corrente e sanitizados com solução refrigerada de hipoclorito de sódio a $200 \mathrm{mg} \mathrm{L}^{-1}$ por $15 \mathrm{~min} \mathrm{e}$ secos sob condições ambientais.

Os frutos de jabuticaba foram divididos em quatro grupos, sendo um composto por frutos sem cobertura e os demais cobertos com soluções filmogênicas preparadas com $2,5 \%$ de fécula de mangarito e concentrações de 10, 20 e 30\% de glicerol. Essas concentrações de plastificante foram selecionadas pelo maior e menor valor médio de Pva e solubilidade em água dos filmes elaborados.

Após o preparo das soluções filmogênicas, que permaneceram em repouso até temperatura próxima à do ambiente $\left(25^{\circ} \mathrm{C}\right)$, os frutos de jabuticaba foram imersos nas soluções filmogênicas por 5 min, suspensos, acomodados em bandejas de poliestireno expandido e deixados até a cobertura secar em temperatura ambiente. A parcela experimental foi composta por 8 frutos.

As bandejas contendo os frutos com as coberturas fixadas e secas foram armazenadas por 4 dias em temperatura ambiente $\left( \pm 25^{\circ} \mathrm{C}\right)$. As bandejas não foram recobertas com outro material de embalagem de forma que as coberturas fossem a única barreira entre os frutos e o meio ambiente. Os tratamentos consistiram de: acondicionamento $1\left(T_{1}\right)$ - Fruto com cobertura a $10 \%$ de glicerol; acondicionamento $2\left(T_{2}\right)$ - Fruto com cobertura a $20 \%$ de glicerol; acondicionamento $3\left(T_{3}\right)$ - Fruto com cobertura a $30 \%$ de glicerol; controle $4\left(T_{4}\right)$ - Fruto sem cobertura.

O tempo de armazenamento variou de 0 a 4 dias, sendo as avaliações efetuadas todos os dias e iniciadas logo após a montagem do experimento. Foram avaliados a perda de massa, a SST/ ATT e o pH dos frutos. As análises foram realizadas em cinco repetições, à temperatura ambiente.

Antes cada análise, os frutos foram lavados com água destilada para remoção das coberturas. Não houve destruição das amostras na avaliação da perda de massa. Para as análises químicas, efetuadas conforme metodologia do Instituto Adolfo Lutz (1985), as amostras foram amassadas e coadas, sem adição de água.

A perda de massa foi avaliada em todos os períodos de armazenamento, utilizando-se balança semi-analítica (Marte, AL 500, Santa Rita do Sapucaí/MG, Brasil) com precisão de 0,001 g, sendo os resultados expressos em porcentagem sobre a massa inicial (Equação 5):

$$
\text { Perda de massa }(\%)=100\left[\frac{\left(\mathrm{m}_{0}-m\right)}{m_{0}}\right]
$$

Em que:

$\mathrm{m}_{0}=$ massa inicial da amostra $(\mathrm{kg}) ; \mathrm{m}=$ massa a cada intervalo de tempo $(\mathrm{kg})$.

Mediu-se o teor de sólidos solúveis totais em refratômetro digital (CETI, Belgium), com precisão de 0,1 e leitura direta por meio da colocação de algumas gotas do suco do fruto de jabuticaba na placa do refratômetro (resultados em graus brix, ${ }^{\circ}$ Brix).

Para a determinação da acidez total titulável utilizou-se solução de hidróxido de sódio (Vetec Química Fina, Rio de Janeiro/RJ, Brasil), a 0,1 $\mathrm{M} \mathrm{L}^{-1} \mathrm{e}$ indicador de fenolftaleína.

A determinação do $\mathrm{pH}$ foi realizada no suco da fruta de jabuticaba (amassada e coada sem adição de água), utilizando-se potenciômetro (TECNAL, TEC3-MP) com precisão de 0,001 e mantendo-se a solução homogeneizada. 
Adotou-se delineamento estatístico inteiramente casualizado (DIC), disposto em esquema fatorial $4 \times 5$, com cinco repetições, em que o primeiro fator correspondeu aos tratamentos: 0 (controle), 10, 20 e 30\% de glicerol e o segundo ao tempo de armazenamento (0, 1, 2, 3 e 4 dias). Cada parcela foi composta por oito frutos.

Usando-se ANOVA, a 5\% de probabilidade, uma equação de segunda ordem foi aplicada para explicar a tendência das variáveis respostas (Y): perda de massa, relação SST/ATT e pH, (equação geral conforme a Equação 4).

Para o desenvolvimento das análises estatísticas e dos gráficos das propriedades dos filmes e conservação pós-colheita empregou-se o software Statistic versão 8.0 (STATSOFT, 2007).

\section{RESULTADOS E DISCUSSÃO}

A aplicação de coberturas biodegradáveis em frutos de jabuticaba torna-se importante para reduzir a taxa respiratória e aumentar sua vida-de-prateleira. Para isso devem ser escolhidos filmes que apresentem baixos valores de permeabilidade ao vapor e solubilidade em água. Os valores médios desses parâmetros, determinados nos filmes biodegradáveis elaborados à base de fécula de mangarito e glicerol, estão registrados na Tabela 2.

TABELA 2 - MÉDIA E DESVIO PADRÃO DA PERMEABILIDADE AO VAPOR DE ÁGUA (Pva) E SOLUBILIDADE EM ÁGUA DAS COBERTURAS BIODEGRADÁVEIS ELABORADOS À BASE DE FÉCULA DE MANGARITO E GLICEROL

\begin{tabular}{l|c|c|c|c}
\hline Tratamentos & Glicerol (\%) & Fécula $(\mathrm{g})$ & Solubilidade & $\begin{array}{l}\text { Pva }\left(\times 10^{-5}\right) \\
{\left[\mathrm{g}(\mathrm{m} \mathrm{s} \mathrm{Pa})^{-1}\right]}\end{array}$ \\
\hline T1 & 12,9 & 1,4 & $3,09 \pm 0,04$ & $3,30 \pm 0,13$ \\
\hline T2 & 12,9 & 3,6 & $3,67 \pm 0,04$ & $3,24 \pm 0,21$ \\
\hline T3 & 27,7 & 1,4 & $3,87 \pm 0,10$ & $3,10 \pm 0,25$ \\
\hline T4 & 27,7 & 3,6 & $2,43 \pm 0,20$ & $3,28 \pm 0,10$ \\
\hline T5 & 9,6 & 2,5 & $2,96 \pm 0,15$ & $3,26 \pm 0,02$ \\
\hline T6 & 30,4 & 2,5 & $2,47 \pm 0,27$ & $3,23 \pm 0,03$ \\
\hline T7 & 20,0 & 1,0 & $3,00 \pm 0,04$ & $3,18 \pm 0,02$ \\
\hline T8 & 20,0 & 4,0 & $3,01 \pm 0,01$ & $3,11 \pm 0,05$ \\
\hline T9 & 20,0 & 2,5 & $3,23 \pm 0,30$ & $3,39 \pm 0,35$ \\
\hline T10 & 20,0 & 2,5 & $3,19 \pm 0,95$ & $3,15 \pm 0,02$ \\
\hline T11 & 20,0 & 2,5 & $3,07 \pm 0,85$ & $3,11 \pm 0,09$ \\
\hline
\end{tabular}

Médias obtidas de duas repetições.

De acordo com Mali et al. (2004), Laohakunjit e Noomhorm (2004) e Müller, Yamashita e Laurindo (2008), a permeabilidade dos filmes biodegradáveis está associada à disponibilidade de grupos hidroxilas para ligação com a água e quanto maior for a disponibilidade maior será a permeabilidade dos mesmos. Kester e Fenema (1989) mencionaram que a presença do plastificante nos filme aumenta a sua hidrofilicidade, proporcionando o movimento das cadeias poliméricas e, consequentemente, facilitando o movimento das moléculas que atravessam o filme.

A variação da PVA observada na Tabela 2 não foi significativa, segundo a ANOVA (Tabela 3). Nenhuma das variáveis independentes ou sua interação afetaram a permeabilidade dos filmes estudados, portanto, todos os filmes apresentaram o mesmo valor de permeabilidade ao vapor de água, em torno de $3,1210^{-5} \mathrm{~g}(\mathrm{~m} \mathrm{~s} \mathrm{~Pa})^{-1}$. 
TABELA 3 - ANÁLISE DE VARIÂNCIA PARA PERMEABILIDADE AO VAPOR DE ÁGUA DE FILMES BIODEGRADÁVEIS À BASE DE FÉCULA DE MANGARITO E GLICEROL

\begin{tabular}{lccccc}
\hline Fator & $\mathrm{GL}$ & $\mathrm{SQ}$ & $\mathrm{QM}$ & $\mathrm{F}$ & Valor- $p$ \\
\hline Glicerol $(\mathrm{G})$ & 1 & 0,009 & 0,009 & $0,323^{\text {n.s. }}$ & 0,579 \\
$\mathrm{G}^{2}$ & 1 & 0,005 & 0,005 & $0,183^{\text {n.s }}$ & 0,676 \\
FÉCULA (A) & 1 & 0,001 & 0,001 & $0,006^{\text {n.s }}$ & 0,942 \\
$\mathrm{~A}^{2}$ & 1 & 0,008 & 0,008 & $0,272^{\text {n.s }}$ & 0,611 \\
G x A & 1 & 0,029 & 0,029 & $1,035^{\text {n.s }}$ & 0,328 \\
Falta de ajuste & 3 & 0,019 & 0,006 & $0,229^{\text {n.s }}$ & 0,874 \\
Erro puro & 13 & 0,363 & 0,028 & & \\
Total & 21 & 0,438 & & & \\
\hline
\end{tabular}

$\mathrm{GL}=$ graus de liberdade; $\mathrm{SQ}=$ soma dos quadrados; $\mathrm{QM}$ = quadrado médio; $\mathrm{F}=$ Teste $\mathrm{F} ;$ Valor- $p$ = probabilidade estatística. n.s $=$ não significativo.

A solubilidade em água constitui importante propriedade das coberturas comestíveis no que se refere às suas aplicações. Coberturas com alta solubilidade podem ser interessantes para embalagens de alimentos desidratados que devam sofrer hidratação prévia ao consumo. Entretanto, muitas aplicações demandam embalagens firmes à água, como no caso de alimentos com alta atividade de água, ou mesmo para se evitar a transpiração de produtos frescos (MONTERREYQUINTERO e SOBRAL, 1998). Sendo assim, observa-se na Tabela 2 dois grupos de filmes com diferentes solubilidades, o primeiro entre 2,43 e $2,96 \%$ e o segundo de 3,0 a 3,87\%. Essas diferenças são confirmadas pela ANOVA (Tabela 4) e a variação de solubilidade ocorre pela interação das variáveis aplicadas $(p<0,01)$, gerando equação polinomial de segunda ordem:

$$
\text { Solubilidade }(\%)=0,444+0,144 \mathrm{G}+1,211 \mathrm{~A}-0,065 \mathrm{G} \mathrm{A}
$$

\section{TABELA 4 - ANÁLISE DE VARIÂNCIA PARA SOLUBILIDADE EM ÁGUA DE FILMES BIODEGRADÁVEIS À BASE DE FÉCULA DE MANGARITO E GLICEROL}

\begin{tabular}{lccccc}
\hline Fator & GL & SQ & QM & F & Valor- $p$ \\
\hline Glicerol (G) & 1 & 0,313 & 0,313 & $2,103^{\text {n.s. }}$ & 0,171 \\
Amido (A) & 1 & 0,211 & 0,211 & $1,421^{\text {n.s. }}$ & 0,255 \\
G x A & 1 & 2,059 & 2,059 & $13,842^{* *}$ & 0,003 \\
Falta de ajuste & 5 & 1,050 & 0,210 & $1,412^{\text {n.s. }}$ & 0,283 \\
Erro puro & 13 & 1,933 & 0,149 & & \\
Total & 21 & 5,529 & & & \\
\hline
\end{tabular}

$\mathrm{GL}=$ graus de liberdade; $\mathrm{SQ}=$ soma dos quadrados; $\mathrm{QM}$ = quadrado médio; $\mathrm{F}=$ Teste $\mathrm{F} ;$ Valor- $p$ = probabilidade estatística. ** = significativo ao nível de $1 \%$ de probabilidade; n.s = não significativo.

A Figura 1 mostra que as máximas solubilidades podem ser alcançadas quando os filmes são elaborados com $1 \%$ de amido e $30 \%$ de glicerol, ou com $4 \%$ de amido e $9 \%$ de glicerol. Já a 
menor solubilidade foi obtida em filmes elaborados com $1 \%$ de amido e $9 \%$ de glicerol e $4 \%$ de amido e $30 \%$ de glicerol. Resultados semelhantes foram encontrados por Tapia-Blacido (2006) em filmes biodegradáveis preparados com amaranto. A elevação da concentração de plastificantes de 0,3 para $0,4 \%$ aumentou a solubilidade dos filmes em 10,6\%. Laohakunjit e Noomhorm (2004), estudando filmes de amido de arroz e glicerol e Mali et al. (2004) filmes de amido de inhame e glicerol, constataram que maior concentração do plastificante eleva a solubilidade dos filmes.

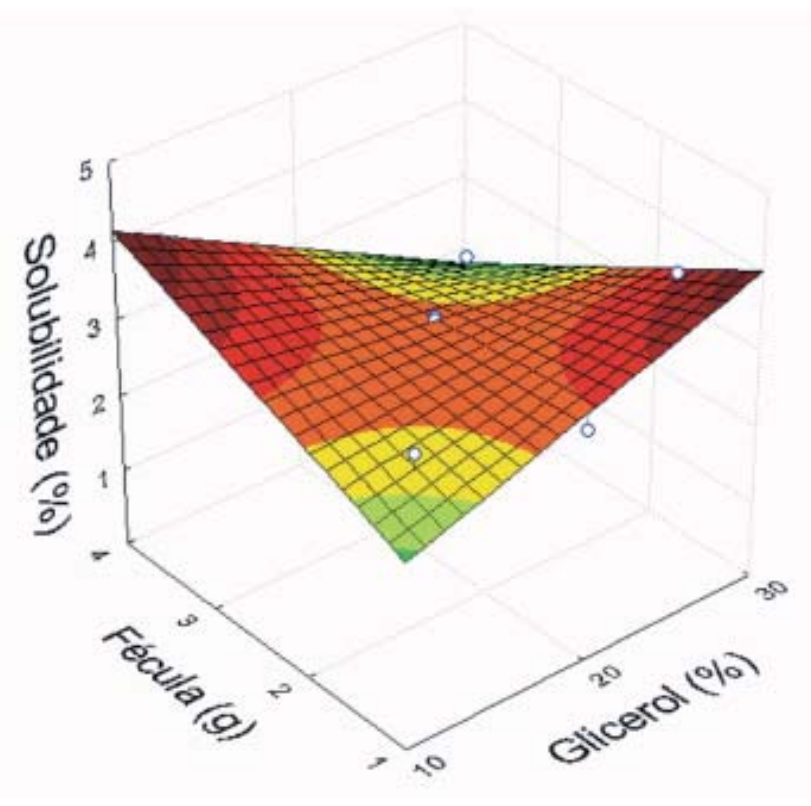

\section{FIGURA 1 - SUPERFÍCIE DE RESPOSTA PARA A SOLUBILIDADE EM ÁGUA DE FILMES BIODEGRADÁVEIS À BASE DE FÉCULA DE MANGARITO E GLICEROL}

Os resultados das análises dos frutos de jabuticaba sem e com cobertura estão representados em forma de gráficos nas Figuras 2 a 4 para perda de massa, SST/ATT e pH, respectivamente. Os gráficos foram gerados após análise de variância e adequação do modelo matemático proposto.

De acordo com a ANOVA (Tabela 5), apenas o efeito linear do glicerol não influenciou a perda de massa dos frutos de jabuticabas sem e com cobertura, indicando que não houve perdas proporcionais ao aumento ou diminuição da quantidade de glicerol adicionada na cobertura. Entretanto, verificou-se influência dessa variável concomitantemente com o tempo de armazenamento como mostrando na Tabela 5. A interação $G \times T$ foi altamente significativa, indicando que o efeito do glicerol depende do efeito do tempo e vice-versa.

A perda de massa da frutas cobertas ou não em função da concentração de glicerol e do tempo de armazenamento foi representada pelo seguinte modelo polinomial:

$$
\text { Perda de massa } \begin{aligned}
(\mathrm{g})= & 48,25+0,01 \mathrm{G}-0,01 \mathrm{G}^{2}-9,64 \mathrm{~T} \\
& +1,02 \mathrm{~T} 2+0,104 \mathrm{GT}
\end{aligned}
$$

Na Figura 2 observam-se poucas diferenças entre as perdas de massa dos frutos com e sem cobertura ao longo do tempo de armazenamento. Esse fato demonstra que o uso das coberturas não foi efetivo no controle da perda de massa e que essa característica independe da concentração do glicerol. 
TABELA 5 - ANÁLISE DE VARIÂNCIA APLICADA A UM MODELO MATEMÁTICO POLINOMIAL PARA PREDIZER A PERDA DE MASSA DOS FRUTOS DE JABUTICABA EM FUNÇÃO DA CONCENTRAÇÃO DE GLICEROL NA COBERTURA DE FÉCULA DE MANGARITO E DO TEMPO DE ARMAZENAMENTO

\begin{tabular}{lcrcc}
\hline Fonte de Variação & GL & \multicolumn{1}{c}{ QM } & F & Valor-p \\
\hline Glicerol (G) & 1 & 0,762 & 0,097 & $0,756^{\text {n.s }}$ \\
G $^{2}$ & 1 & 49,337 & 6,306 & $0,014^{*}$ \\
Tempo (T) & 1 & 3172,858 & 405,521 & $\leq 0,01^{* *}$ \\
T $^{2}$ & 1 & 292,333 & 37,363 & $\leq 0,01^{* *}$ \\
G x T & 1 & 271,921 & 34,754 & $\leq 0,01^{* *}$ \\
Erro puro & 94 & 7,824 & - & - \\
TOTAL & 99 & - & - & - \\
\hline
\end{tabular}

$\mathrm{GL}=$ graus de liberdade; $\mathrm{QM}=$ quadrado médio; $\mathrm{F}=$ teste $\mathrm{F} ;$ Valor $-\mathrm{p}=$ valor da probabilidade.

${ }^{*}$ significativo a $1 \%$ de probabilidade; ${ }^{*}$ significativo a $5 \%$ de probabilidade; n.s = não significativo.

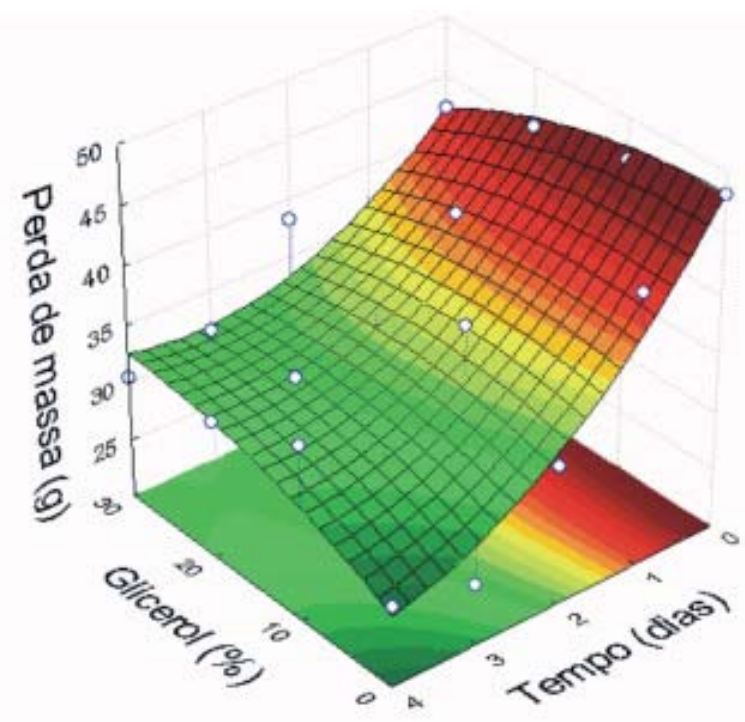

FIGURA 2 - SUPERFÍCIE DE RESPOSTA PARA PERDA DE MASSA DOS FRUTOS DE JABUTICABA EM FUNÇÃO DA CONCENTRAÇÃO DE GLICEROL ADICIONADA À COBERTURA DE FÉCULA DE MANGARITO E DO TEMPO DE ARMAZENAMENTO

As coberturas contendo $10 \%$ de glicerol foram efetivas somente na redução da perda de massa das jabuticabas mantidas à temperatura ambiente. Entretanto não se pode dizer o mesmo para as frutas sem cobertura e aquelas revestidas com os tratamentos $T_{2}$ e $T_{3}$, verificando-se murchamento no decorrer do armazenamento. Essas observações concordam com os resultados de Oliveira (1996), cuja película a $5 \%$ retardou a perda de massa de goiaba, quando comparados com a testemunha. 
As coberturas de fécula de mangarito e glicerol não foram efetivas, considerando a maioria dos resultados demonstrados. Tal fato se deve, provavelmente, à baixa barreira ao vapor de água conferida por filmes comestíveis feitos à base de amido (GALLO et al., 2000). O sucesso do revestimento comestível para atender as necessidades específicas de frutas frescas depende fortemente da sua propriedade de barreira à umidade, que por sua vez depende da estrutura dos polímeros que formam o revestimento e da condição de armazenamento. Dessa forma, provavelmente não houve formação de barreira efetiva para controlar a redução da transpiração dos frutos.

A maioria dos materiais de polissacarídeos à base de fécula é hidrofílico, deixando de controlar a perda de umidade dos alimentos. Por outro lado, os materiais de revestimento hidrofóbicos, como poliésteres de sacarose, fornecem melhores barreiras à umidade (KESTER e FENNEMA, 1986; DEBEAUFORT, QUEZADA-GALLO e VOILLEY, 1998; MILLER, UPADHYAYA e KROCHTA, 1998; KROCHTA, 2002). Acredita-se que a utilização de maior teor de fécula na solução filmogênica formadora das coberturas poderia aumentar sua espessura e a barreira ao vapor de água, provocando menor perda de massa pelos frutos.

A perda de água de produtos armazenados não resulta apenas em perda de massa, mas também na diminuição de qualidade (LEMOS, 2006). Chitarra e Chitarra (2005) afirmaram que alguma perda de massa pode ser tolerada, mas aquelas responsáveis por murchamento devem ser evitadas. No quinto dia, a perda de massa dos frutos de jabuticabas provocou murchamento em todos os tratamentos.

Lemos et al. (2007), em estudo sobre a perda de massa de pimentões 'Magali R' acondicionados à temperatura ambiente e sob refrigeração, verificaram que o uso de recobrimentos à base de $2 \%$ de fécula de mandioca não foi efetivo na redução dessa característica. A mesma tendência foi certificada por Botrel et al. (2007) em alho minimamente processado com revestimentos comestíveis de fécula de mandioca, não havendo diferença estatística para o atributo perda de massa.

Oliveira e Cereda (2003) observaram que o acréscimo de cera em coberturas de fécula de mandioca proporcionou maior eficiência na redução da perda de massa de pêssegos armazenados à temperatura ambiente.

O fruto de jabuticaba apresenta casca muito fina que confere pouca proteção à perda de umidade, o que pode ter contribuído para os altos valores de perda de massa encontrados e a redução de sua vida após a colheita.

Recobrimentos comestíveis de fécula podem contribuir para aumentar o período de conservação dos frutos de jabuticabas, porém deve-se buscar formulações que minimizem a perda de matéria fresca, sem restringir excessivamente as trocas gasosas.

O teor médio de SST dos frutos de jabuticabas (17,91 ${ }^{\circ}$ Brix) assemelhou-se aos valores obtidos por Asquieri et al. (1997), Brunini et al. (2004); Sato e Cunha (2007); Lima et al. (2008) e Oliveira et al. (2008) em trabalhos realizados com frutos de jabuticabas.

Os frutos de jabuticaba com maiores valores de SST, segundo Silva et al. (2003) implicam em menor adição de açúcares, menor tempo de evaporação da água e menor gasto de energia. É importante destacar que o fruto de jabuticaba tem alto teor de SST, superior a outros frutos, como goiaba 'Pedro Sato' com 9,05 'Brix (HOJO et al., 2007) e a pitanga com 11,47 ${ }^{\circ}$ Brix (LOPES, MATTIETTO e MENEZES., 2005).

A análise de variância para estudar os efeitos que causam a concentração do glicerol adicionado á cobertura de fécula de mangarito e o tempo de armazenamento na variação da relação de SST/ATT, consta na Tabela 6. As frutas de jabuticabas cobertas com solução de fécula de mangarito e glicerol não diferiram do controle, nem entre si, indicando que o material testado não influenciou essa característica. Tal fato pode ser justificado pela alta permeabilidade ao vapor de água dos filmes. 
TABELA 6 - ANÁLISE DE VARIÂNCIA APLICADA A UM MODELO MATEMÁTICO POLINOMIAL PARA PREDIZER A RELAÇÃO SSTIATT DOS FRUTOS DE JABUTICABA EM FUNÇÃO DA CONCENTRAÇÃO DE GLICEROL NA COBERTURA DE FÉCULA DE MANGARITO E DO TEMPO DE ARMAZENAMENTO

\begin{tabular}{lcccc}
\hline Fonte de Variação & GL & QM & \multicolumn{1}{c}{$F$} & $P$ \\
\hline Glicerol $(G)$ & 1 & 0,496 & 0,285 & $0,595^{\text {n.s }}$ \\
G $^{2}$ & 1 & 0,770 & 0,442 & $0,508^{\text {n.s }}$ \\
Tempo (T) & 1 & 13,654 & 7,838 & $0,006^{* *}$ \\
T $^{2}$ & 1 & 27,059 & 15,533 & $\leq 0,001^{* *}$ \\
G X T & 1 & 18,537 & 10,641 & $0,002^{* *}$ \\
Erro puro & 94 & 1,742 & - & - \\
TOTAL & 99 & - & - & - \\
\hline
\end{tabular}

$\mathrm{GL}=$ graus de liberdade; $\mathrm{QM}=$ quadrado médio; $\mathrm{F}=$ teste $\mathrm{F} ;$ Valor $-p=$ valor da probabilidade. ${ }^{*}$ significativo a $1 \%$ de probabilidade; ${ }^{* *}$ significativo a $5 \%$ de probabilidade; n.s = não significativo.

Atribuiu-se a variação da relação SST/ATT ao tempo de armazenamento e à interação $\mathrm{G} x$ $T$, cujos efeitos foram altamente significativos $(p \leq 0,01)$. O modelo matemático que representa essa variação se expressa da seguinte forma:

$$
\text { SST/ATT }=8,91-0,57 \mathrm{~T}+0,31 \mathrm{~T}^{2}-0,3 \mathrm{G} \mathrm{T}
$$

A Equação (8) gerou o gráfico da Figura 3, que indica a ocorrência de altos valores de SST/ ATT durante o armazenamento das amostras em condição ambiental. Esse aumento no teor de sólidos solúveis totais confere degradação de polissacarídeos no decorrer do processo respiratório dos frutos (CHITARRA e CHITARRA, 1990).

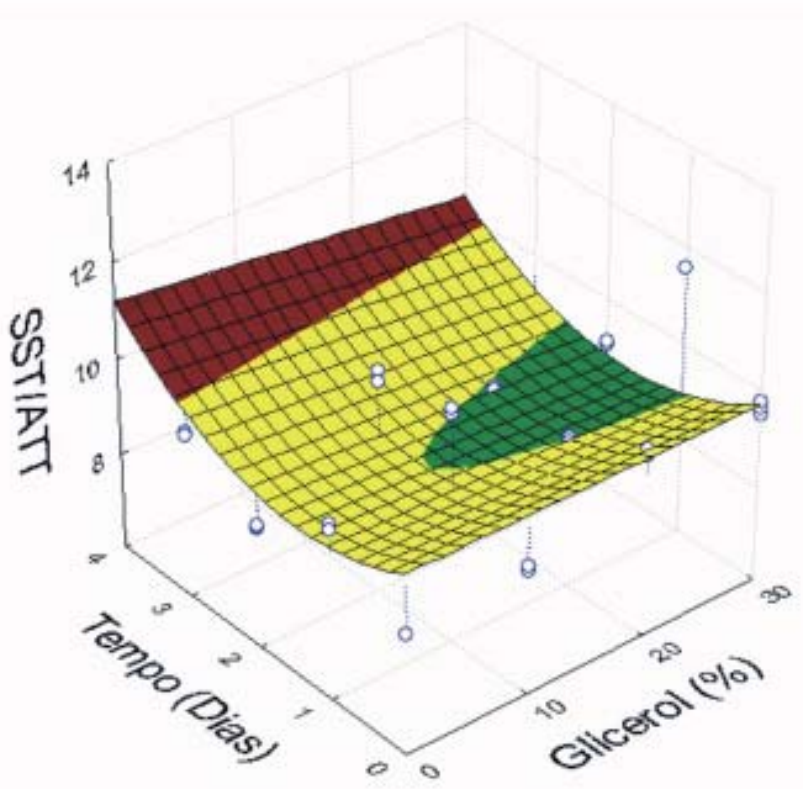

FIGURA 3 - SUPERFÍCIE DE RESPOSTA PARA OS VALORES DA RELAÇÃO SÓLIDOS SOLÚVEIS TOTAISIACIDEZ TITULÁVEL TOTAL (SSTIATT) DOS FRUTOS DE JABUTICABA EM FUNÇÃO DA CONCENTRAÇÃO DE GLICEROL ADICIONADA À COBERTURA DE FÉCULA DE MANGARITO E DO TEMPO DE ARMAZENAMENTO 
Segundo Chitarra e Chitarra (2005), durante o armazenamento, o fruto tende a maturação e os valores de SST aumentam devido à hidrólise de polissacarídeos em açúcares simples e os valores de ATT diminuem por meio da oxidação do ácido no ciclo de Krebs. Sendo assim, a relação SST/ATT deve gradativamente aumentar com o período de armazenamento. Porém, Olivas e Barbosa-Cánovas (2005) afirmaram que a quantidade de água existente no fruto deve ser sempre considerada nos valores relacionados de SST e ATT. A quantidade de água perdida causa aumento nos valores de SST e ATT, podendo-se chegar a resultado incorreto.

$\mathrm{O}$ valor médio de $\mathrm{pH}$ dos frutos de jabuticabas $(3,41)$ aproximou-se dos valores de $\mathrm{pH}$ encontrados por Oliveira, Brunini e Salandi (2003) e Geócze (2010) para o mesmo fruto (2,91 a 3,72 e 3,22 a 3,50 , respectivamente).

A variação do $\mathrm{pH}$ seguiu o mesmo comportamento da ATT, diminuindo com o decorrer do tempo de armazenamento frutos (Tabela 7).

\section{TABELA 7 - ANÁLISE DE VARIÂNCIA APLICADA A UM MODELO MATEMÁTICO POLINOMIAL PARA PREDIZER OS VALORES DE PH DOS FRUTOS DE JABUTICABA, EM FUNÇÃO DA CONCENTRAÇÃO DE GLICEROL NA COBERTURA DE FÉCULA DE MANGARITO E DO TEMPO DE ARMAZENAMENTO}

\begin{tabular}{lccrc}
\hline Fonte de Variação & GL & QM & \multicolumn{2}{c}{$\mathrm{P}$} \\
\hline Glicerol (G) & 1 & 0,059 & 4,790 & $0,031^{*}$ \\
G $^{2}$ & 1 & 0,057 & 4,584 & $0,035^{*}$ \\
Tempo (T) & 1 & 1,797 & 145,455 & $\leq 0,001^{* *}$ \\
T$^{2}$ & 1 & 1,443 & 116,766 & $\leq 0,001^{* *}$ \\
G x T & 1 & 0,029 & 2,308 & $0,132^{\text {n.s }}$ \\
Erro puro & 94 & 0,029 & - & - \\
TOTAL & 99 & & - & - \\
\hline
\end{tabular}

$\mathrm{GL}=$ graus de liberdade; $\mathrm{QM}=$ quadrado médio; $\mathrm{F}=$ teste $\mathrm{F} ;=$ Valor $-p=$ valor da probabilidade.

${ }^{*}$ significativo a $1 \%$ de probabilidade; ${ }^{* *}$ significativo a $5 \%$ de probabilidade; n.s = não significativo.

Descreve-se o modelo matemático que melhor se adequou à variação do $\mathrm{pH}$ dos frutos de jabuticaba (sem ou com cobertura) em função do glicerol contido na cobertura e o tempo de armazenamentos dos frutos, como:

$$
\mathrm{pH}=3,29+0,01 \mathrm{G}-0,0002 \mathrm{G}^{2}-0,18 \mathrm{~T}+0,07 \mathrm{~T}^{2}
$$

Não houve interação entre os efeitos do glicerol contido nas coberturas e o tempo de armazenamento. Observou-se redução no pH dos quatro tratamentos ao longo do tempo de armazenamento, o que pode estar associado aos altos valores de perda de massa das amostras (Figura 4). A perda de água nos frutos pode ter sido decorrente do aumento na concentração de ácidos orgânicos, causando diminuição do $\mathrm{pH}$. Valores semelhantes foram encontrados por Pereira et al. (2000) em frutos de oito clones de jabuticabeiras. 


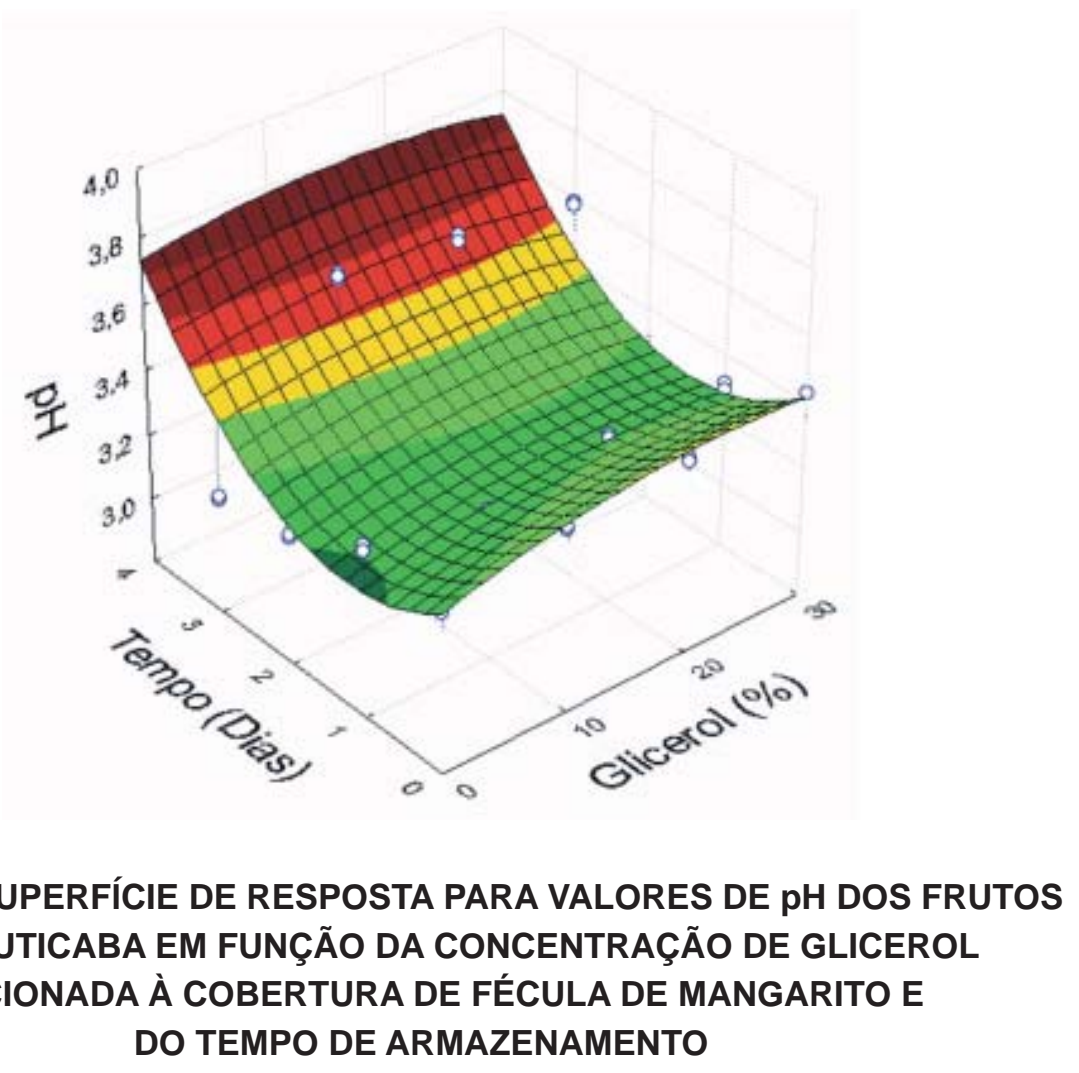

\section{FIGURA 4 - SUPERFÍCIE DE RESPOSTA PARA VALORES DE PH DOS FRUTOS DE JABUTICABA EM FUNÇÃO DA CONCENTRAÇÃO DE GLICEROL ADICIONADA À COBERTURA DE FÉCULA DE MANGARITO E DO TEMPO DE ARMAZENAMENTO}

\section{CONCLUSÃO}

De acordo com os resultados obtidos no presente trabalho, os filmes de fécula de mangarito e glicerol apresentaram solubilidade entre 2,43 e $3,87 \%$ e permeabilidade de $3,10 \times 10^{-5} \mathrm{e}$ $\left.3,39 \times 10^{-5} \mathrm{~g} \mathrm{(m} \mathrm{s} \mathrm{Pa}\right)^{-1}$.

Maior concentração de plastificante aumentou a permeabilidade ao vapor de água e a solubilidade. As soluções filmogênicas mostraram-se muito hidrofílicas. Não foi verificada diferença significativa para a permeabilidade ao vapor de água dos filmes. Equação de segunda ordem foi a mais adequada para descrever a variação da solubilidade.

A utilização das coberturas de fécula de mangarito e glicerol não foi eficiente para retardar o metabolismo dos frutos de jabuticabas armazenados em temperatura ambiente. Não foi verificada diferença significativa entre as frutas de jabuticaba com e sem cobertura, constatando-se apenas interação entre os fatores glicerol e tempo.

\section{ABSTRACT \\ DEVELOPMENT AND CHARACTERIZATION OF EDIBLE FILMS OF MANGARITO (Xanthosoma mafaffa Schott) AND ITS APPLICATION ON JABUTICABA FRUITS}

The present work aimed at using the Mangarito rhizomes starch for use as coatings for post-harvest fruit jabuticaba. The quality of coverage was assessed by the permeability and solubility of the biodegradable films and also by physical and chemical parameters of fruits determined due to certain storage time. The biodegradable films were prepared from Mangarito rhizomes starch and glycerol in concentrations ranging from 1.44 and $3.56 \mathrm{~g}, 12.93$ and $27.70 \%$ respectively. The biodegradable coatings were prepared with $2.5 \%$ starch and glycerol concentrations ranging from 10 to $30 \%$. Was used the central composite experimental design, type star, with five replicates at center point and four axial to check the effect of variation in starch and plasticizer on the solubility and permeability of the films, aiming at selecting the best coverage by response surface methodology. For post-harvest conservation was adopted a completely randomized design arranged 
in a $4 \times 5$ factorial scheme, with five replicates, the first factor corresponded to the treatments: 0 to $30 \%$ glycerol and the second to storage time (0-4 days). The increase in glycerol concentration in the solution filmogenic collaborated with the increased permeability and solubility of the films. The biodegradable coatings were not effective in controlling physical and chemical transformations of jabuticaba fruits as the loss of mass, the relationship between $\mathrm{pH}$ and total soluble solids and titratable acidity, not being observed significant difference between uncoated or coated fruits with different coatings.

KEY-WORDS: Xanthosoma mafaffa Schott; STARCH; JABUTICABA FRUIT; EDIBLE FILMS.

\section{REFERÊNCIAS}

1 ASCHERI, D. P. R.; ANDRADE, C. T.; CARVALHO, C. W. P.; ASCHERI, J. L. R. Obtenção de farinhas mistas prégelatinizadas a partir de arroz e bagaço de jabuticaba: efeito das variáveis de extrusão nas propriedades de pasta. Boletim do Centro de Pesquisa e Processamento de Alimentos, Curitiba, v. 24, n. 1, p. 115-144, 2006.

2 ASQUIERI, E. R.; CANDIDO, M. A.; DAMIANI, C.; ASSIS, E. M. Fabricación de vino blanco y tinto de jabuticaba (Myrciaria jabuticaba Berg) utilizando la pulpa y la cáscara respectivamente. Alimentaria, Madrid, n. 355, p. 97-109, 1997.

3 BOTREL, D. A.; SOARES, N. de F. F.; GERALDINE, R. M.; PEREIRA, R. M.; FONTES, E. A. F. Qualidade de alho (Allium sativum) minimamente processado envolvido com revestimento comestível microbiano. Ciência e Tecnologia de Alimentos, Campinas, v. 27, n. 1, p. 32-38, 2007.

4 BOX, G. E. P.; HUNTER, W. G.; HUNTER, J. S. Statistics for experimenters: an introduction to design, data analysis, and modo building. New York: Wiley and Sons, 1978. 653 p.

5 BRUNINI, M. A.; OLIVEIRA, A. L.; SALANDINI, C. A. R.; BAZZO, F. R. Influência de embalagens e temperatura no armazenamento de jabuticabas (Myrciaria jabuticaba (vell) berg) cv 'sabará'. Ciência e Tecnologia de Alimentos, Campinas, v. 24, n. 3, p. 378-383, 2004.

6 CHARNET, R.; FREIRE, C. A. L.; CHARNET, E. M. R.; BONVINO, H. Análise de modelos de regressão linear com aplicações. Campinas: Editora da UNICAMP, 1999. 356 p.

7 CHITARRA, M. I. F.; CHITARRA, A. B. Pós-colheita de frutas e hortaliças: fisiologia e manuseio. Lavras: ESAL, FAEPE, 1990. $320 \mathrm{p}$.

8 CHITARRA, M. I. F.; CHITARRA, A. B. Pós-colheita de frutas e hortaliças: fisiologia e manuseio. 2. ed. Lavras: UFLA, 2005. $783 \mathrm{p}$.

9 COCHRAN, W. G.; COX, G. M. Experimental designs. $2^{\text {nd }}$ ed. New York: John Wiley, 1964. 617 p.

10 COSTA, C. A. da; RAMOS, S. J; ALVES, D. S.; FERNADES, L. A.; SAMPAIO, R. A.; MARTINS, E. R. Nutrição mineral do mangarito num Latossolo Vermelho Amarelo. Horticultura Brasileira, Brasília, v. 26, n. 1, p.102-106, 2008.

11 DEBEAUFORT, F; QUEZADA-GALLO J. A.; VOILLEY, A. Edible films and coatings: tomorrows packagings: a review. Critical Reviews in Food Science and Nutrition, Philadelphia, v. 38, n. 4, p. 299-313, 1998.

12 FERREIRA, M. D.; FRANCO, A. T. O.; NOGUEIRA, M. F. M.; ALMEIDA, R. V. C. de; TAVARES, M. Avaliação da etapa da colheita em tomates de mesa cv. Débora. Brazilian Journal of Food Technology, Campinas, v. 7, n. 2, p. 173-178, 2004.

13 GALLO, J. A. Q.; DEBEAUFORT, F.; CALLEGARIN, F.; VOILLEY, A. Lipidic hydrophobic, physical state and distribution effects on the properties of emulsion-based films. Journal of Membrane Science, Amsterdam, v. 180, n. 1, p. 37- 46, 2000.

14 GEÖCZE, A. C. Influência da preparação de licor de jabuticaba (Myrciaria jaboticaba Vell berg) no teor de compostos fenólicos. 2007. 81 p. Dissertação (Mestrado em Ciência de Alimentos) - Faculdade de Farmácia, Belo Horizonte, 2007.

15 GONTARD, N.; GUILBERT, S.; CUQ, J. L. Edible wheat gluten films: influence of the main process variables on film properties using response surface methodology. Journal of Food Science, Chicago, v. 57, n. 1, p. 190-199, 1992

16 HOJO, E. T. D.; CARDOSO, A. D.; HOJO, R. H.; VILAS BOAS, E. V. de B.; ALVARENGA, M. A. R. Uso de películas de fécula de mandioca e PVC na conservação pós-colheita de pimentão. Ciência e Agrotecnologia, Lavras, v. 31, n. 1, p. 184-190, 2007.

17 INSTITUTO ADOLFO LUTZ. Normas analíticas: métodos químicos e físicos para análises de alimentos. 3. ed. São Paulo, 1985. v. 1. 
18 KESTER, J. J.; FENNEMA, O. R. An edible film of lipids and cellulose ethers: barrier properties to moisture vapor transmission and structural evaluation. Journal of Food Science, Chicago, v. 54, n. 6, p. 1383-1389, 1989.

19 KHURI, A. I.; CORNELL, J. A. Response surfaces. New York: Marcel Dekker, 1987.

$20 \mathrm{KROCHTA}$, J. M. Proteins as raw materials for films and coatings: definitions, current status, and opportunities. In: GENNADIOS, A. (ed). Protein-based films and coatings. Boca Raton: CRC Press, 2002. p. 1-41.

21 LAOHAKUNJIT, N.; NOOMHORM, A. Effect of plasticizers on mechanical and barrier properties of rice starch film. Starch/ Stärke, Weinheim, v. 56, p. 348-356, 2004

22 LEITE, G. L. D.; SILVA, F. W. S.; JESUS, F. M.; COSTA, C. A.; GUANABENS, R. E. M.; GUSMÃO, C. A. G. Efeito da adubação orgânica, espaçamento e tamanho de rizoma-semente sobre artrópodes em mangarito Xanthosoma mafaffa Schott. Arquivos do Instituto Biológico, São Paulo, v. 74, n. 4, p. 343-348, 2007.

23 LEMOS, O. L. Utilização de biofilmes comestíveis na conservação pós-colheita do pimentão 'Magali R'. 2006. 115 p. Dissertação (Mestrado em Agronomia) - Universidade Estadual do Sudoeste da Bahia, Vitória da Conquista, 2006.

24 LEMOS, O. L.; REBOUÇAS, T. N. H.; SÃO JOSÉ, A. R.; VILA, M. T. R.; SILVA, K. S. Utilização de biofilme comestível na conservação de pimentão ‘MagaliR'em duas condições dearmazenamento. Bragantia, Campinas, v. 66, n. 4, p. 693-699, 2007.

25 LIMA, A. de. J. B.; CORRÊA, A. D.; ALVES, A. P. C.; ABREU, C. M. P.; BARRO, A. M. D. Caracterização química do fruto jabuticaba (Myrciaria cauliflora Berg) e de suas frações. Archivos Latinoamericanos de Nutrición, Caracas, v. 58, n. 4, p. 416-421, 2008.

26 LOPES, A. S.; MATTIETTO, R. de A.; MENEZES, H. C. de. Estabilidade da polpa de pitanga sob congelamento. Ciência e Tecnologia de Alimentos, Campinas, v. 25, n. 3, p. 553- 559, 2005.

27 MALI, S.; GRossmANN, M. V. E.; GARCÍA, M. A.; MARTINO, M. M.; ZARITZKY, N. E. Barrier, mechanical and optical properties of plasticized yam starch films. Carbohydrate Polymers, Barking, v. 56, n. 2, p. 129-135, 2004.

28 MILLER, K. S.; UPADHYAYA, S. K.; KROCHTA, J. M. Permeability of d-limonene in whey protein films. Journal of Food Science, Chicago, v. 63, p. 244-247, 1998.

29 MONTEIRO, D. A; PERESSIN, V. A. Efeito do tamanho do rizoma-semente, da época e do local de plantio, na produção de rizomas de mangará. Bragantia, Campinas, v. 56, n.1, p. 155-161, 1997.

30 MONTERREY-QUINTERO, E. S.; SOBRAL, P. J. A. Preparo de proteínas miofibrilares de tilápia-do-nilo para a elaboração de biofilmes: solubilidade das proteínas em função do pH. In: CONGRESSO BRASILEIRO DE CIÊNCIAE TECNOLOGIA DE ALIMENTOS, 16., 1998, Rio de Janeiro. Anais... Rio de Janeiro: Sociedade Brasileira de Ciência e Tecnologia de Alimentos, 1998. p. 2086-2089.

31 MULLER, C. C. O.; YAMASHITA, F.; LAURINDO, J. B. Evaluation of the effects of glycerol and sorbitol concentration and water activity on the water barrier properties of cassava starch films through a solubility approach. Carbohydrate Polymers, Barking, v. 72, p. 82-87, 2008.

32 OLIVAS, G. I.; BARBOSA-CÁNOVAS, G. V. Edible coatings for fresh-cut fruits. Critical Reviews in Food Science and Nutrition, Philadelphia, v. 45, p. 657-670, 2005.

33 OLIVEIRA, M. A. Utilização de película de fécula de mandioca como alternativa à cera na conservação póscolheita de frutos de Goiaba (Psidium guajava). 1996. 73 p. Dissertação (Mestrado em Ciências) - Escola Superior de Agricultura "Luiz de Queiroz", Universidade de São Paulo, Piracicaba, 1996.

34 OlIVEIRA, A. L.; NETO, E. A. B.; FENERICH, E. J.; ALONSO, C. O.; AZEVEDO, J. S. A.; NETO, P. O. Efeito da aplicação pré-colheita de cálcio na qualidade dos frutos de jabuticaba. In: CONGRESSO BRASILEIRO DE FRUTICULTURA, 20., E ANNUAL MEETING OF THE INTERAMERICAN SOCIETY FOR TROPICAL HORTICULTURE, 54 ${ }^{\text {th }}$, 2008, Vitória. Anais... Vitória: DCM/Incaper, 2008. CD-ROM

35 OLIVEIRA, A. L; BRUNINI, M. A. SALANDI, C. A. R. Caracterização tecnológica de jabuticabas 'Sabará' provenientes de diferentes regiões de cultivo. Revista Brasileira de Friticultura, Jabuticabal, v. 25, n. 3, p. 397-400, 2003.

36 OLIVEIRA, M. A. de; CEREDA, M. P. Pós-colheita de pêssegos (Prunus pérsica L. Bastsch) revestidos com filmes a base de amido como alternativa à cera comercial. Ciência e Tecnologia de Alimentos, Campinas, v. 23, p. 28-33, 2003.

37 PEREIRA, M. C. T.; SALOMÃO, L. C. C.; MOTA, W. F.; VIEIRA, G. Atributos físicos e químicos de frutos de oito clones de jabuticabeiras. Revista Brasileira de Fruticultura, Jaboticabal, v. 22, n. Especial, p.16-21, 2000.

38 SATO, A. C. K.; CUNHA, R. L. Influência da temperatura no comportamento reológico da polpa de jabuticaba. Ciência Tecnológica de Alimentos, Campinas, v.27, n.4, p. 890-896, 2007. 
39 SILVA, M. M.; CORREAA, A. D.; SANTOS, C. D.; ABREU, C. M. P de. Antinutrientes da fruta-de-lobo (Solanum lycocarpum St. Hil) em diferentes estágios de amadurecimento. In: CONGRESSO DE INICIAÇÃO CIENTÍFICA DA ESAL, 16., 2003, Lavras. Anais... Lavras: UFLA, 2003. p. 229.

40 STATSOFT INC. Statistica (data analysis software system). Version 8.0. Tulsa, 2007. CD-ROM.

41 TÁPIA-BLÁCIDO, D. R. Filmes à base de derivados do amaranto para uso em alimentos. 2006. 351 p. (Doutorado em Engenharia de Alimentos) - Universidade Estadual de Campinas, Campinas, 2006.

42 UTERNOEHL, B.; NUNES JR., O. Agricultura Mbya-Guarani na conservação da biodiversidade: a partir da aldeia marágatu-Imarui/SC. Revista Brasileira de Agroecologia, Porto Alegre, v. 1, n.1, p. 397-401, 2006. 